\title{
Comparison of CMM and Micro-CT Volumetric Analysis of Polyethylene Tibial Knee Inserts in Total Knee Replacement
}

\author{
Wei Jiang $\mathbb{D}^{1},{ }^{1}$ Cuicui Ji, ${ }^{2}$ Huaxing Xiao, ${ }^{1}$ Zhongmin Jin, $^{3}$ and Yuntian Dai ${ }^{1}$ \\ ${ }^{1}$ School of Mechanical and Automobile Engineering, Changzhou Institute of Technology, Changzhou, Jiangsu 213032, China \\ ${ }^{2}$ School of Mechanical and Electrical Engineering, Hohai University, Changzhou, Jiangsu 213022, China \\ ${ }^{3}$ Leeds Joint School, Southwest Jiaotong University, Chengdu, Sichuan 611756, China
}

Correspondence should be addressed to Wei Jiang; jwei@czust.edu.cn

Received 29 December 2017; Revised 22 March 2018; Accepted 22 April 2018; Published 19 June 2018

Academic Editor: Qi Shen

Copyright ( 92018 Wei Jiang et al. This is an open access article distributed under the Creative Commons Attribution License, which permits unrestricted use, distribution, and reproduction in any medium, provided the original work is properly cited.

Ultrahigh molecular weight polyethylene (UHMWPE) bearings are used widely in orthopaedic joint replacement as a prominent material for improving the longevity, which is dramatically influenced by wear of polyethylene. Polyethylene tibial knee components from knee simulators under two different input conditions were analyzed using CMM and Micro-CT measurement techniques for volumetric loss with gravimetric measurement as reference. Based on the coordinates and image slices obtained, the surface curve fitting and image digitization methodology were used for the creation of nominal "original" surface in the case of no prewear data provided. The comparison results indicate that gravimetric remains the gold standard and the CMM measurement took less time and had better precision, accuracy, and repeatability compared to Micro-CT measurement technique.

\section{Introduction}

As an outstanding material for orthopaedic joint replacement, polyethylene can provide excellent abrasion resistance, low friction, high impact resistance, a self-lubricating surface, insignificant water absorption, good chemical resistance, high-energy absorption, and no temperature sensitivity in the human biological environment [1]. Ultrahigh molecular weight polyethylene (UHMWPE) components are being widely used in total knee replacement (TKR) as a bearing surface to improve the longevity, if well designed and properly implanted, products made of this material can function for more than fifteen years or even longer. Currently, the major issue in TKR is the wear of polyethylene and results in more than 16\% failure of TKR [2]. Thus, wear measurement is very important for accurate determination of wear rate and volume loss in total knee replacements. The most standard method is gravimetric analysis $[3,4]$. However, the fundamental issue with gravimetric measurement is that it needs a reference from which to calculate the wear. In addition, gravimetric measurement has limitations such as inclusion of wear debris in the polyethylene component, transfer of materials into the tibial tray, changes in mass of the polyethylene due to fluid uptake, and the cementation of the components [5], which may result in inaccurate volumetric determination from gravimetric analysis [6]. Since it is necessary to have a reference measurement for gravimetric analysis, it is not possible to use this method for retrieved samples. Therefore, to avoid these disadvantages, advanced volumetric measurement techniques such as coordinate measuring machine (CMM) [7] and micro computed topography (Micro-CT) $[8,9]$ have been used to determine the volume change of polyethylene in hip, knee, and spinal replacement devices and validated for accuracy and repeatability. The aim of this study was to compare CMM (Legex 322, Mitotoyo) and Micro-CT 100 (Scanco Medical, Busserdorf, Switzerland) measurement techniques for volumetric measurement of knee simulator specimen using gravimetric measurement of volume loss as reference.

\section{Materials and Methods}

A total of 12 UHMWPE tibial knee components (DePuy, UK), which were tested in six station knee simulators (University of Leeds, UK), were used for measurement. The wear tests were carried out by a colleague within the research 
institution (C. Brockett) in 2009 and 2011 [10]. Two input profiles were used for the specimens, one is the ISO setup and the other is a standard CR150. Both CMM and Micro$\mathrm{CT}$ measurement techniques were applied to obtain the three-dimensional (3-D) surface coordinates and images slices, respectively. In addition, the images slices acquired via Micro-CT were then transferred into coordinates with the help of image digitization. Therefore, the 3-D surface coordinates acquired from CMM and Micro-CT were analyzed volumetrically to investigate the comparison of those measurement techniques. Since prewear data such as original drawings and CAD models are not always available to determine the volume loss of tibial knee components, the original surface needs to be reconstructed. In this study, a 5th order polynomial curve surface fitting algorithm (1) was applied for the generation of original 3-D surface on the basis of undeformed region, which proved its effectiveness and accuracy based on simulated volume removal and computational tests (maximum errors equal to $0.2 \mathrm{~mm}^{3}$ and $1.1 \mathrm{~mm}^{3}$, resp.) [11]. Prior to the surface fitting process, the wear region should be distinguished and removed from the database for accurate original surface reconstruction, which was considered as prewear data for volumetric analysis. As demonstrated in Figure 1, the wear region was determined according to the difference of $Z$ value between two adjacent coordinates, it can be judged to be worn out when the difference is greater than $0.1 \mathrm{~mm}$. It is worth noting that some clearly wrong coordinates need to be removed for accurate wear region identification. The methodologies of CMM and Micro-CT for volumetric measurements are detailed below.

$$
\begin{aligned}
f(x, y)= & P 00+P 10 x+P 01 y+P 20 x^{2}+P 11 x y+P 02 y^{2} \\
& +P 30 x^{3}+P 21 x^{2} y+P 12 x y^{2}+P 03 y^{2}+P 40 x^{4} \\
& +P 31 x^{3} y+P 22 x^{2} y^{2}+P 13 x y^{3}+P 04 y^{4}+P 50 x^{5} \\
& +P 41 x^{4} y+P 32 x^{3} y^{2}+P 23 x^{2} y^{3}+P 14 x y^{4}+P 05 y^{5},
\end{aligned}
$$

where $P i j$ are parameters in polynomial surface fitting algorithm, $i$ is the degree in $x$, and $j$ is the degree in $y$.

2.1. CMM. For CMM measurement, Trigene (MediChem International Ltd., Seven Oaks, UK) was used to clean the specimen before measurement. After removing from the knee simulator, the polyethylene inserts were cleaned using detergent water then soaked in $1 \%$ Trigene solution for 30 minutes to remove all visible serum and contaminants from the surface. Then, the inserts were soaked in isopropanol solution (Fisher Scientific, Loughborough, UK) mixed with water (70\% isopropanol:30\% water) and placed in an ultrasonic bath (VWR Labshop, IL, USA) for 10 minutes (IMBE simulator test protocol, Leeds University, UK). Afterwards, the components were stored in the weighing room, which is temperature and humidity controlled $\left(21^{\circ} \mathrm{C}\right.$ and $40 \%$, resp.) and allowed to stabilize for a period of 48 hours. The change in mass was assessed using the AT 201 balance (Mettler Toledo Inc., Columbus, Ohio, USA), and the volumetric loss was calculated using the following equation, taking the

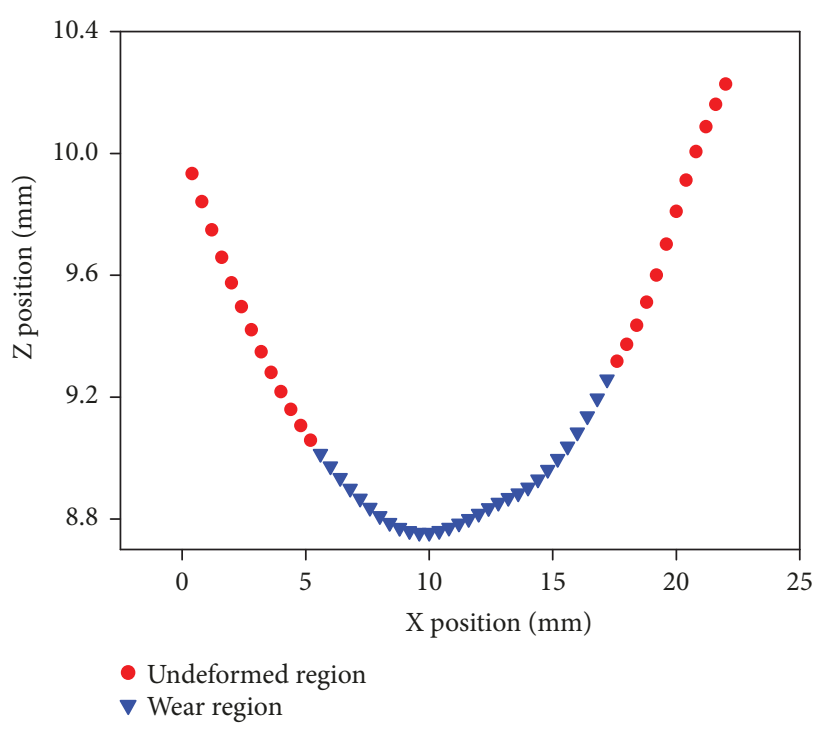

FIGURE 1: Identification of undeformed region.

density of polyethylene as $0.931 \mathrm{~g} / \mathrm{mm}^{3}$ [12]. The samples were measured using CMM (Legex 322, Mitutoyo, UK) with known wear volume determined using gravimetric analysis. It is important to note that the scan interval could influence the volumetric determination dramatically. As a result, an investigation of the influence of the CMM scan interval $(0.1 \mathrm{~mm}, 0.2 \mathrm{~mm}, 0.5 \mathrm{~mm}, 1.0 \mathrm{~mm}, 1.5 \mathrm{~mm}$, and $2.0 \mathrm{~mm})$ was performed using an unworn tibial knee insert and the results indicate that with the increasing of CMM scan interval, the points decreased from 31,133 to 90 and the time consumed decreased from more than 8 hours to nearly 2 minutes, which result in the increase of volume difference from $0.1 \mathrm{~mm}^{3}$ to $8.1 \mathrm{~mm}^{3}$ (Figure 2). Therefore, $0.2 \mathrm{~mm}$ scan interval was used in this study to maintain the equilibrium between accuracy and time consumed. As illustrated in Figure 3, since there was no initial surface data provided, the coordinates of each condyle surface were measured and surface curve fitting methodology was used for volumetric analysis based on the reconstructed "original" surface [13]. The results obtained were compared against gravimetric measurements.

2.2. Micro-CT. For Mico-CT measurement, an accurate threshold was the key parameter for volumetric analysis using Micro-CT (Scanco Medical, Busserdorf, Switzerland). There are two methods to determine the threshold: one is based on gravimetric analysis and the other is reference to a known volume cylindrical reference specimen. Known volumetric difference from gravimetric measurement was essential for the first method to calculate the amount of wear in Micro-CT. However, the specimens before wear were not available. Hence, the latter method was used in this study to determine the threshold based on a cylindrical reference specimen with same material and known volume $\left(936 \mathrm{~mm}^{3}\right)$. The in-built software image processing language (IPL) was applied to determine the threshold as 665. With the obtained threshold, two-dimensional greyscale image slices were transferred from original ISQ file and stored in 


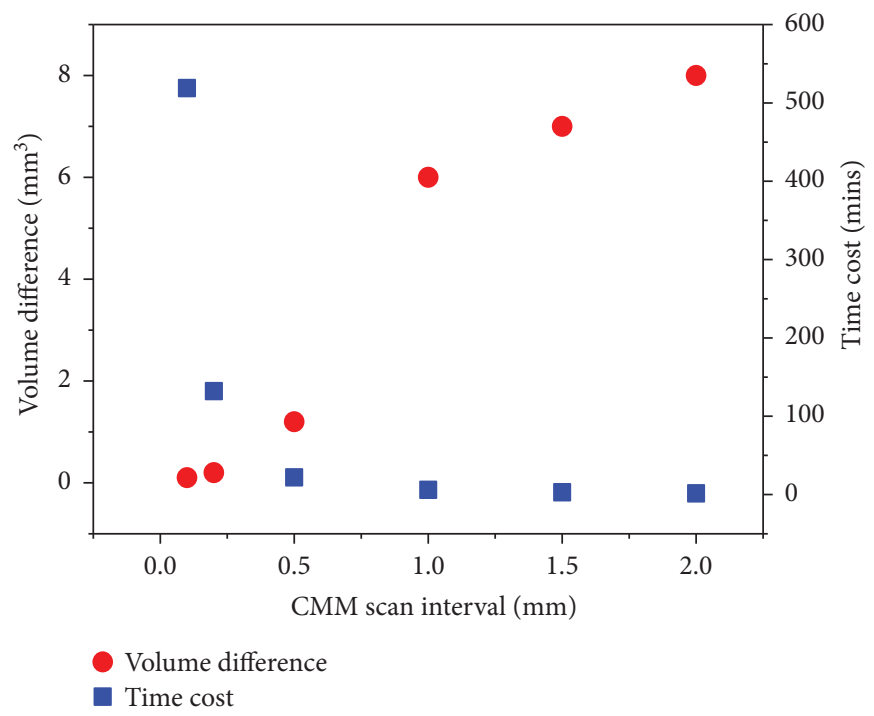

Figure 2: The influence of CMM scan interval on volume difference.

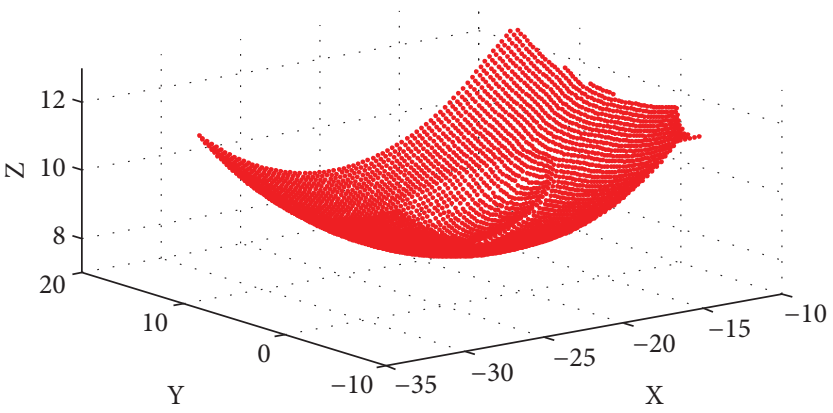

(a) Coordinates captured from a worn surface of left condyle

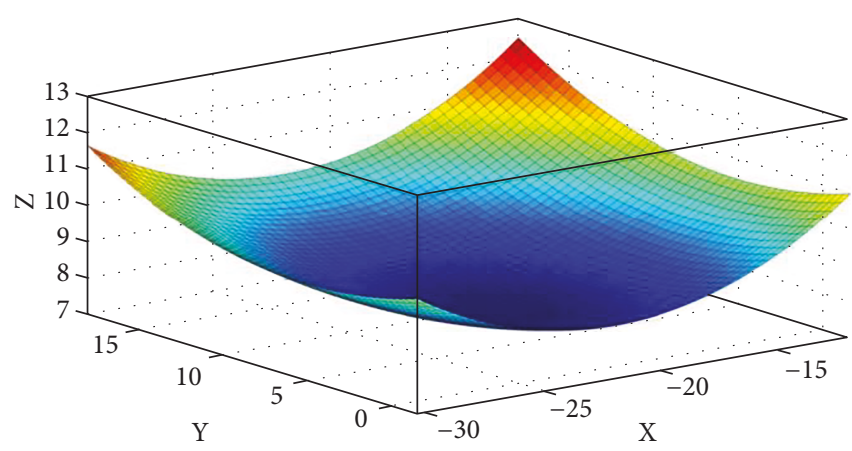

(b) Reconstructed original surface

FIGURE 3: Surface coordinates obtained using CMM and reconstructed original surface for volumetric assessment.

the form of TIFF images and local iterative fitting method was used for 3-D reconstruction. Based on the image digitization method [13], the surface coordinates of the specimens were obtained for determination of the volume loss of the polyethylene specimens using surface curve fitting methodology. All measurements were performed three times on each specimen to determine an average volume change and $95 \%$ confidence limits (CL) calculated, to determine the repeatability of the measurement method. Table 1 demonstrates the parameters of Micro-CT scanner 100 during scanning, and the schematic diagram of Micro-CT procedure is illustrated in Figure 4.

\section{Results and Discussion}

The comparison of CMM and Micro-CT methodologies against gravimetric is shown in Table 2. At lower volumes, the volume calculated using CMM and Micro-CT measurement was close to gravimetric analysis. At higher volumes, both CMM and Micro-CT measurement overestimated wear compared to gravimetric volume and the volume loss determined using Micro-CT was higher than CMM. The measurement time cost for the CMM and Micro-CT was approximately 40 minutes and 90 minutes per scan, respectively. It can be seen from Figure 5 that both CMM and Micro-CT measurement appear to be an overestimate compared to gravimetric measurement. Gravimetric measurement methodology is the standard method used in the determination of volume loss $[3,4]$. However, fluid absorption and metallic debris inclusion may affect the measurement. Methodologies with greater accuracy and repeatability without these limitations are required. CMM and Micro-CT measurement techniques were used in this project to determine the volume loss of polyethylene tibial knee specimens and compared against gravimetric analysis, which was considered as the reference. However, it should be noticed that gravimetric measurement does not include creep whereas CMM and Micro-CT do as they are geometry-based measurement techniques. Besides, backside wear will be included in gravimetric measurement but not in CMM/Micro-CT in this project since geometry changes only in the left and right condyles of the tibial knee components were considered.

In this study, both CMM and Micro-CT analysis were based on the coordinates of the articulating surface. For CMM measurement, the coordinates were obtained directly from the scanning probe and stored on the PC in the form 
TABLE 1: Scanning parameters of Micro-CT100.

\begin{tabular}{lccccc}
\hline Peak voltage & Current & Specimen holder & Integration time & Resolution & Gaussian \\
\hline $70 \mathrm{kVp}$ & $114 \mu \mathrm{A}$ & $74 \mathrm{~mm}$ & $300 \mathrm{~ms}$ & $1024 \times 1024$ & $0.8,1$ \\
\hline
\end{tabular}

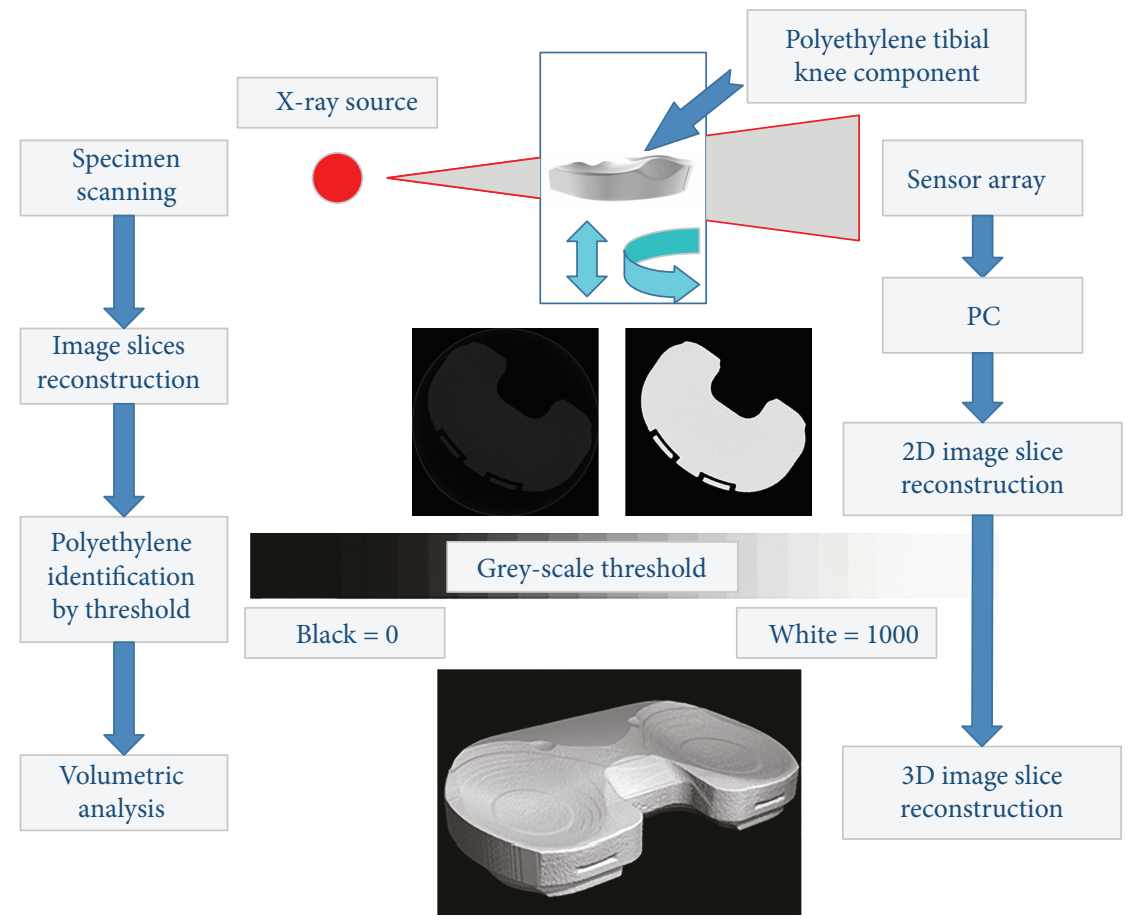

FIGURE 4: Schematic diagram of Micro-CT procedure.

TABLe 2: CMM and Micro-CT volumetric measurement results.

\begin{tabular}{lccc}
\hline Specimen & $\begin{array}{c}\text { Gravimetric } \\
\left(\mathrm{mm}^{3}\right)\end{array}$ & $\begin{array}{c}\text { CMM } \\
\left(\mathrm{mm}^{3}\right)\end{array}$ & $\begin{array}{c}\text { Micro-CT } \\
\left(\mathrm{mm}^{3}\right)\end{array}$ \\
\hline Specimen 1 & 20.12 & 22.85 & 23.31 \\
Specimen 2 & 18.41 & 19.52 & 21.15 \\
Specimen 3 & $\mathbf{9 . 1 2}$ & $\mathbf{1 0 . 0 5}$ & $\mathbf{1 1 . 1 6}$ \\
Specimen 4 & 9.96 & 10.87 & 11.86 \\
Specimen 5 & 14.90 & 16.78 & 16.18 \\
Specimen 6 & 11.02 & 12.16 & 13.35 \\
Specimen 7 & 45.74 & 49.39 & 51.56 \\
Specimen 8 & 38.87 & 41.76 & 45.47 \\
Specimen 9 & $\mathbf{4 9 . 9 2}$ & $\mathbf{5 2 . 5 7}$ & $\mathbf{5 5 . 9 5}$ \\
Specimen 10 & 47.44 & 51.26 & 53.68 \\
Specimen 11 & 40.06 & 43.65 & 42.54 \\
Specimen 12 & 37.47 & 39.61 & 41.39 \\
\hline
\end{tabular}

of txt file for analysis. For Micro-CT measurement, the two-dimensional greyscale images were transferred into coordinates for volumetric analysis. The threshold is a key parameter in Micro-CT scanning and may vary depending on the type and manufacturer of the polyethylene tibial knee components [14]. It has been reported that differences in the measured volumetric wear may be due to errors in thresholding and in the scanner which affect the intensity of the image
[15]. In order to obtain accurate surface geometry, a known volume reference specimen with the same material and $\mathrm{X}$-ray absorption as the specimens measured was used to determine the threshold, which was the most important parameter for image digitization. However, even with the determined threshold, the average repeatability of MicroCT $\left( \pm 3.26 \mathrm{~mm}^{3}\right)$ was still higher than that of gravimetric measurement $\left( \pm 0.02 \mathrm{~mm}^{3}\right)$. The CMM $\left( \pm 0.89 \mathrm{~mm}^{3}\right)$ had better repeatability than Micro-CT, but still higher than gravimetric. Concordance correlation coefficients (CCC) [16] were used to evaluate the two measurement techniques against gravimetric measurement. As demonstrated in Figures 6(a) and 6(b), both CMM and Micro-CT measurement indicated higher wear compared to gravimetric analysis. With the help of web tool created by Lin and validated by $\mathrm{Yu}$ [17], the CCC, precision (Pearson correlation coefficient measuring how closely the observations deviate from the best-fit line), and accuracy (the closeness of the observations and target values in terms of both means and variances) coefficients were calculated, which are illustrated in Table 3. CMM (CCC $=0.990$ and 0.979 for statistic and 95\% CL, resp.) measurement shows a higher concordance than Micro-CT measurement ( $C C C=0.975$ and 0.947 for statistic and 95\% CL, resp.) versus the gravimetric analysis, this is more likely due to the thresholding in Micro-CT and image digitization from two-dimensional greyscale image slices. Besides, both precision (1.000 and 


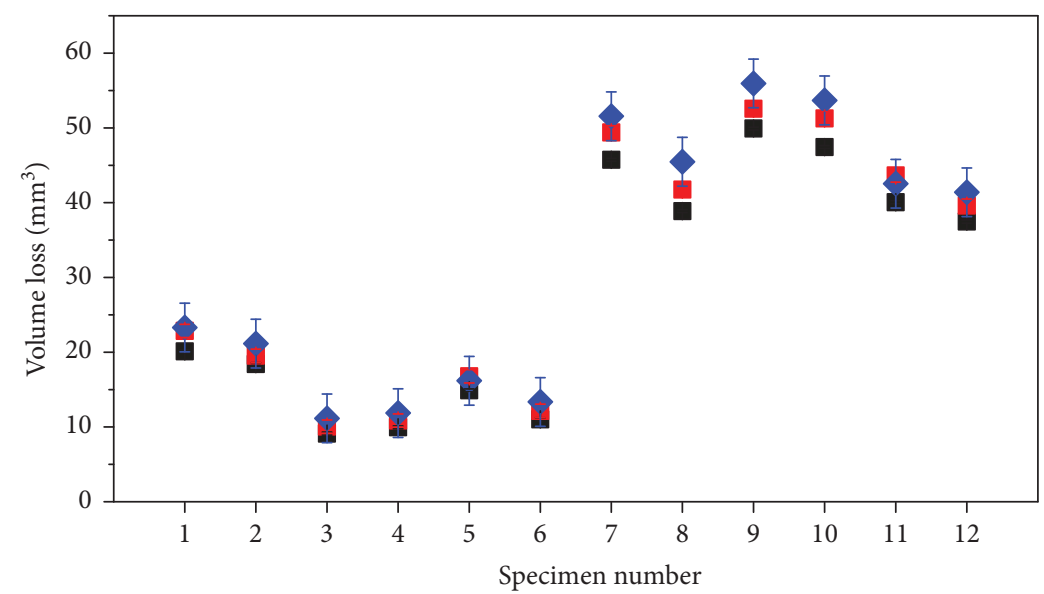

Gravimetric measurement

CMM measurement

Micro-CT measurement

Figure 5: CMM and Micro-CT measurement results of laboratory specimens with 95\% confidence limit.

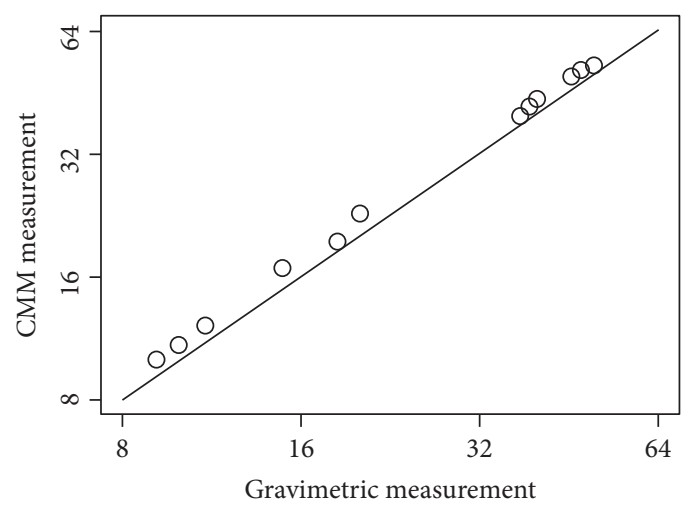

(a) $\mathrm{CMM}$

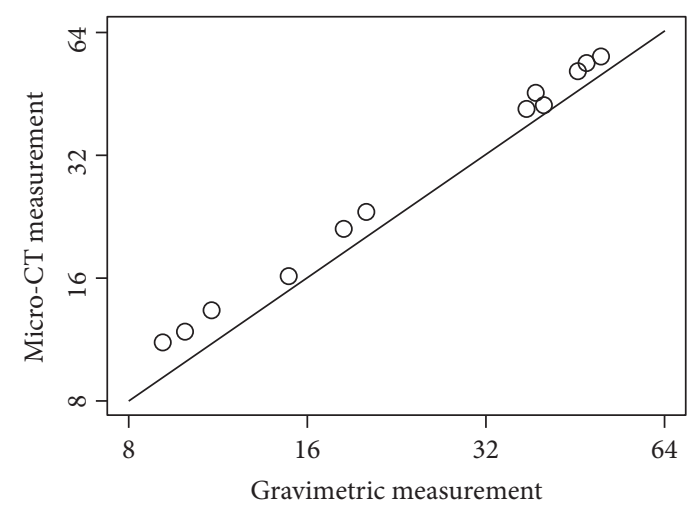

(b) Micro-CT

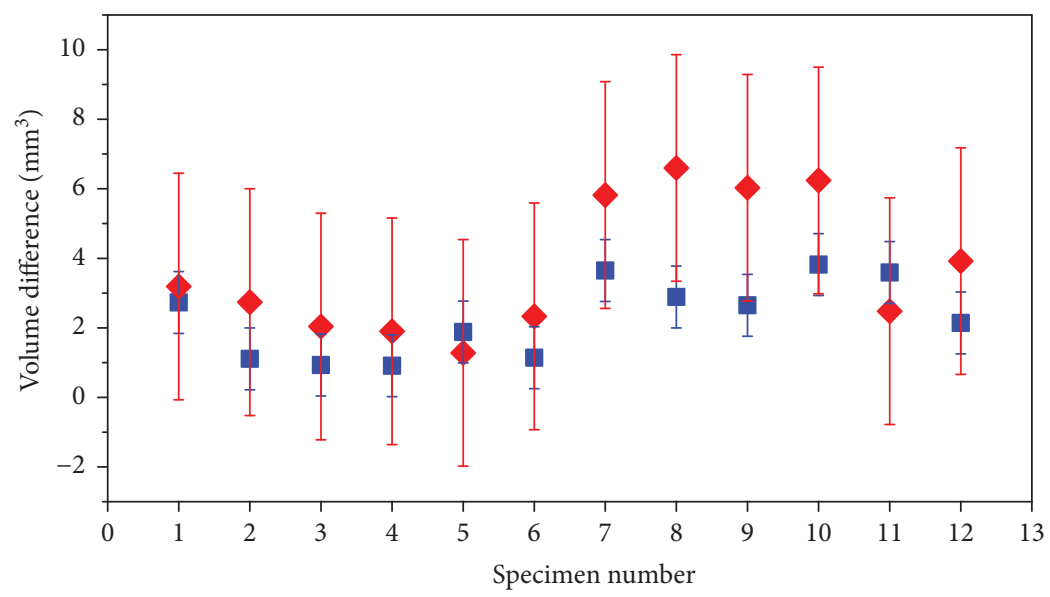

CMM measurement

Micro-CT measurement

(c) Volume difference of CMM and Micro-CT measurement

FIgure 6: Comparison of CMM and Micro-CT volumetric analysis against gravimetric measurement.

0.999 for statistic and 95\% CL, resp.) and accuracy (0.999 and 0.996 for statistic and 95\% CL, resp.) of CMM measurement were greater than Micro-CT. In addition, as demonstrated in
Figure 6(c), the minimum and maximum volume difference of CMM analysis was $0.91 \mathrm{~mm}^{3}$ and $3.82 \mathrm{~mm}^{3}$. The corresponding volume difference of Micro-CT analysis was 
TABle 3: CMM and Micro-CT measurement versus gravimetric measurement.

\begin{tabular}{|c|c|c|c|}
\hline \multicolumn{4}{|c|}{ CMM measurement versus gravimetric measurement } \\
\hline & $\mathrm{CCC}$ & Precision & Accuracy \\
\hline Statistic & 0.990 & 1.000 & 0.991 \\
\hline $95 \% \mathrm{CL}$ & 0.979 & 0.999 & 0.980 \\
\hline \multicolumn{4}{|c|}{ Micro-CT measurement versus gravimetric measurement } \\
\hline & CCC & Precision & Accuracy \\
\hline Statistic & 0.975 & 0.999 & 0.976 \\
\hline $95 \% \mathrm{CL}$ & 0.947 & 0.996 & 0.949 \\
\hline
\end{tabular}

$1.90 \mathrm{~mm}^{3}$ and $6.60 \mathrm{~mm}^{3}$. These studies indicated that CMM had better precision and accuracy compared to Micro-CT analysis. Nevertheless, the voxel size used in the Micro-CT analysis was $79 \mu \mathrm{m}$ and studies show that the volumetric measurements would be underestimated with the increasing of voxel size [18], lower voxel size and higher resolution could result in better volumetric determination compared to CMM measurement with scan interval of $0.2 \mathrm{~mm}$, which needs further analysis.

Since the IPL software can determine the volume of the specimen, Micro-CT can also be used to determine the volume loss with reference data provided $[19,20]$, which is like gravimetric measurements. However, for retrievals or cases similar to the present study, due to the absence of prewear data, the reference surface is not available, surface curve fitting methodology based on CMM coordinates and image digitization would be more appropriate to determine the volume loss. Gravimetric measurement was used as reference in this study to investigate the surface curve fitting methodology based on CMM and Micro-CT measurement techniques. Both CMM and Micro-CT can be used for retrievals when no prewear data exists; however, more specimens should be measured to investigate the applicability and accuracy of the method at higher volume loss, especially when no unworn area was available as reference for surface curve fitting.

\section{Conclusions}

In summary, gravimetric volume analysis remains the most accurate and easiest method to determine the volume loss of polyethylene specimens. However, gravimetric analysis cannot be used at all for retrieval studies, which is an important area of interest in clinical performance. CMM and Micro-CT are alternative measurement techniques used for volume loss determination. In the study, scan interval of $0.2 \mathrm{~mm}$ was adopted to balance the accuracy and time cost for CMM measurement and a known volume cylindrical reference with the same material was used for accurate greyscale threshold determination. Since no prewear data was available for reference, a 5 th polynomial surface curve fitting algorithm was performed to reconstruct a nominal "original" surface based on the unworn surface. In addition, both CMM and Micro-CT techniques could determine the back-side wear of tibial knee inserts, which is not available for gravimetric measurement. The CMM measurement took less time and had better precision, accuracy, and repeatability compared to Micro-CT measurement technique.

\section{Conflicts of Interest}

The authors declare that there is no conflict of interest regarding the publication of this paper.

\section{Acknowledgments}

This research is part of Wei Jiang's $\mathrm{PhD}$ thesis in the University of Leeds and was supported by the EPSRC, the Centre of Excellence in Medical Engineering funded by the Wellcome Trust and by the NIHR Leeds Musculoskeletal Biomedical Research Unit (LMBRU), the Applied Basic Research Programs of Science and Technology Commission Foundation of Jiangsu Province (BK20150256), the National Natural Science Foundation of China (51505126), the Prospective Project of Jiangsu Science and Technology Department (BY2016031-02), and Scientific Research Foundation of Changzhou Institute of Technology (YN1512, YN1626).

\section{References}

[1] S. M. Kurtz, UHMWPE Biomaterials Handbook: Ultra High Molecular Weight Polyethylene in Total Joint Replacement and Medical Devices, Academic Press, 2009.

[2] B. C. Carr and T. Goswami, "Knee implants - review of models and biomechanics," Materials \& Design, vol. 30, no. 2, pp. 398413, 2009.

[3] S. Affatato, B. Bordini, C. Fagnano, P. Taddei, A. Tinti, and A. Toni, "Effects of the sterilisation method on the wear of UHMWPE acetabular cups tested in a hip joint simulator," Biomaterials, vol. 23, no. 6, pp. 1439-1446, 2002.

[4] D. D. D'Lima, J. C. Hermida, P. C. Chen, and C. W. Colwell, "Polyethylene cross-linking by two different methods reduces acetabular liner wear in a hip joint wear simulator," Journal of Orthopaedic Research, vol. 21, no. 5, pp. 761-766, 2003.

[5] P. Bills, L. Blunt, and X. Jiang, "Development of a technique for accurately determining clinical wear in explanted total hip replacements," Wear, vol. 263, no. 7-12, pp. 1133-1137, 2007.

[6] L. A. Blunt, P. J. Bills, X. Q. Jiang, and G. Chakrabarty, "Improvement in the assessment of wear of total knee replacements using coordinate-measuring machine techniques," Proceedings of the Institution of Mechanical Engineers, Part H: Journal of Engineering in Medicine, vol. 222, no. 3, pp. 309-318, 2008.

[7] M. Spinelli, S. Carmignato, S. Affatato, and M. Viceconti, "CMM-based procedure for polyethylene non-congruous unicompartmental knee prosthesis wear assessment," Wear, vol. 267, no. 5-8, pp. 753-756, 2009.

[8] M. G. Teeter, D. D. R. Naudie, K. D. Charron, and D. W. Holdsworth, "Three-dimensional surface deviation maps for analysis of retrieved polyethylene acetabular liners using micro-computed tomography," Journal of Arthroplasty, vol. 25, no. 2, pp. 330-332, 2010.

[9] S. Affatato, F. Zanini, and S. Carmignato, "Quantification of wear and deformation in different configurations of polyethylene acetabular cups using micro X-ray computed tomography," Materials, vol. 10, no. 3, p. 259, 2017. 
[10] C. Brockett, A. Abdelgaied, T. Haythornthwaite, C. Hardaker, J. Fisher, and L. Jennings, "The influence of simulator input conditions on the wear of total knee replacements: An experimental and computational study," Proceedings of the Institution of Mechanical Engineers, Part H: Journal of Engineering in Medicine, vol. 230, no. 5, pp. 429-439, 2016.

[11] W. Jiang, C. Ji, Z. Jin, and Y. Dai, "CMM-based volumetric assessment methodology for polyethylene tibial knee inserts in total knee replacement," Applied Bionics and Biomechanics, vol. 2018, Article ID 9846293, 6 pages, 2018.

[12] C. L. Brockett, L. M. Jennings, and J. Fisher, "The wear of fixed and mobile bearing unicompartmental knee replacements," Proceedings of the Institution of Mechanical Engineers, Part H: Journal of Engineering in Medicine, vol. 225, no. 5, pp. 511-519, 2011.

[13] J. Wei, J. Fisher, Z. Jin, K. W. Ruth, and C. L. Brockett, Wear Measurement of Polyethylene Components in Total the Knee Replacement, [PhD Thesis], University of Leeds, Leeds, LS2 9JT, UK, 2014.

[14] N. Otsu, "A threshold selection method from gray-level histograms," IEEE Transactions on Systems, Man, and Cybernetics, vol. 9, no. 1, pp. 62-66, 1979.

[15] V. Kinzel, M. Scaddan, B. Bradley, and D. Shakespeare, "Varus/valgus alignment of the femur in total knee arthroplasty. Can accuracy be improved by pre-operative CT scanning?," The Knee, vol. 11, no. 3, pp. 197-201, 2004.

[16] L. Lin, "Overview of agreement statistics for medical devices," Journal of Biopharmaceutical Statistics, vol. 18, no. 1, pp. 126144, 2007.

[17] L. Lin, A. S. Hedayat, and W. Wu, Statistical Tools for Measuring Agreement, Springer, 2012.

[18] D. Maret, N. Telmon, O. A. Peters et al., "Effect of voxel size on the accuracy of 3D reconstructions with cone beam CT," Dentomaxillofacial Radiology, vol. 41, no. 8, pp. 649-655, 2012.

[19] M. G. Teeter, J. S. Milner, D. D. R. Naudie, and S. J. MacDonald, "Surface extraction can provide a reference for micro-CT analysis of retrieved total knee implants," The Knee, vol. 21, no. 4, pp. 801-805, 2014.

[20] M. G. Teeter, D. D. R. Naudie, D. D. McErlain et al., "In vitro quantification of wear in tibial inserts using microcomputed tomography," Clinical Orthopaedics and Related Research ${ }^{\circledR}$, vol. 469, no. 1, pp. 107-112, 2011. 


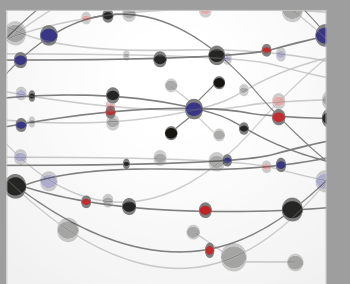

The Scientific World Journal
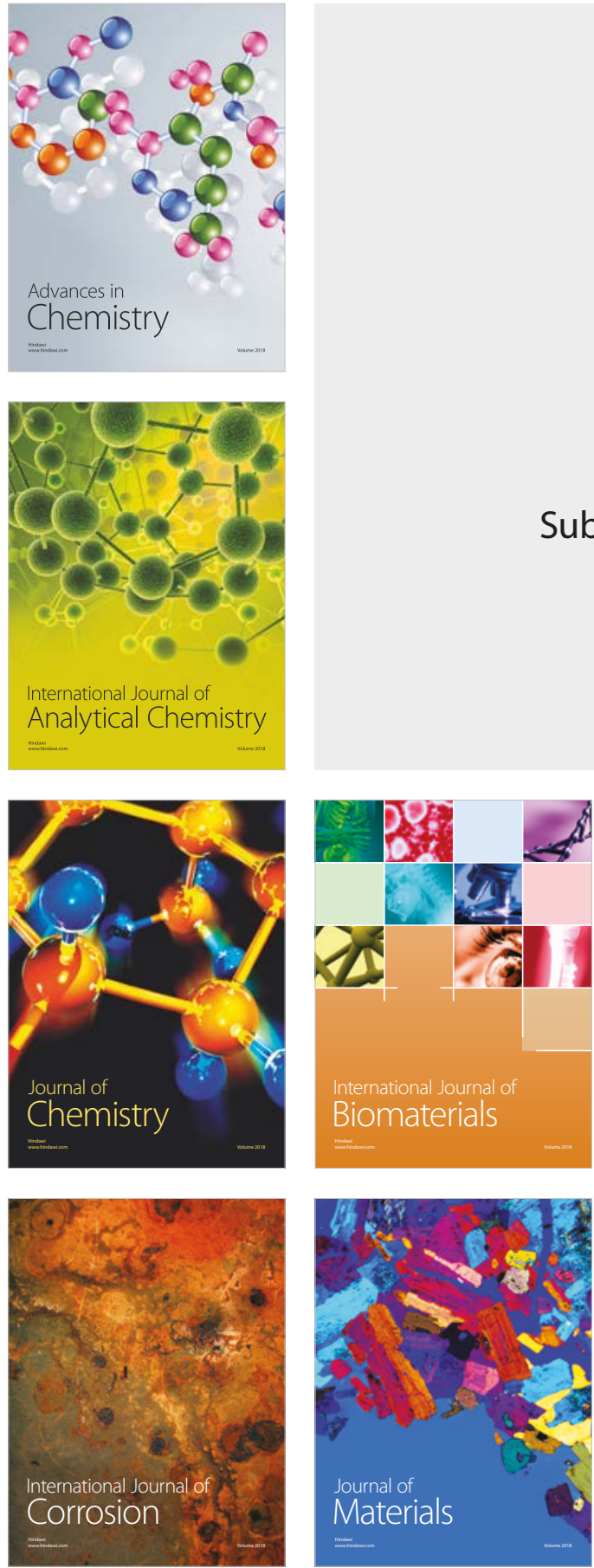

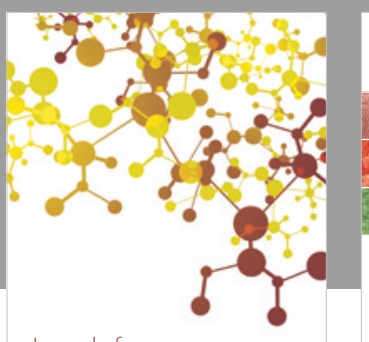

Journal of

Applied Chemistry
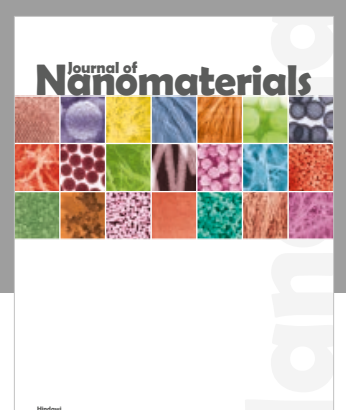

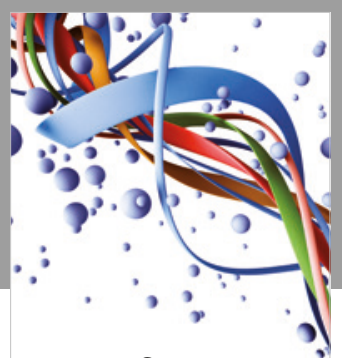

Scientifica

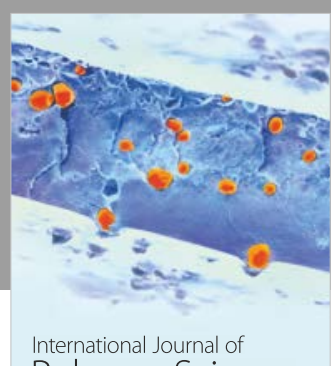

Polymer Science

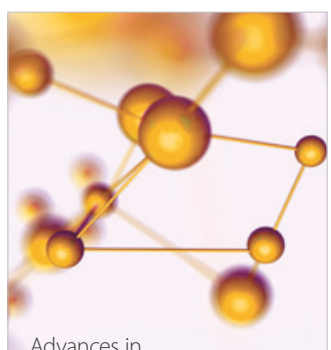

Physical Chemistry
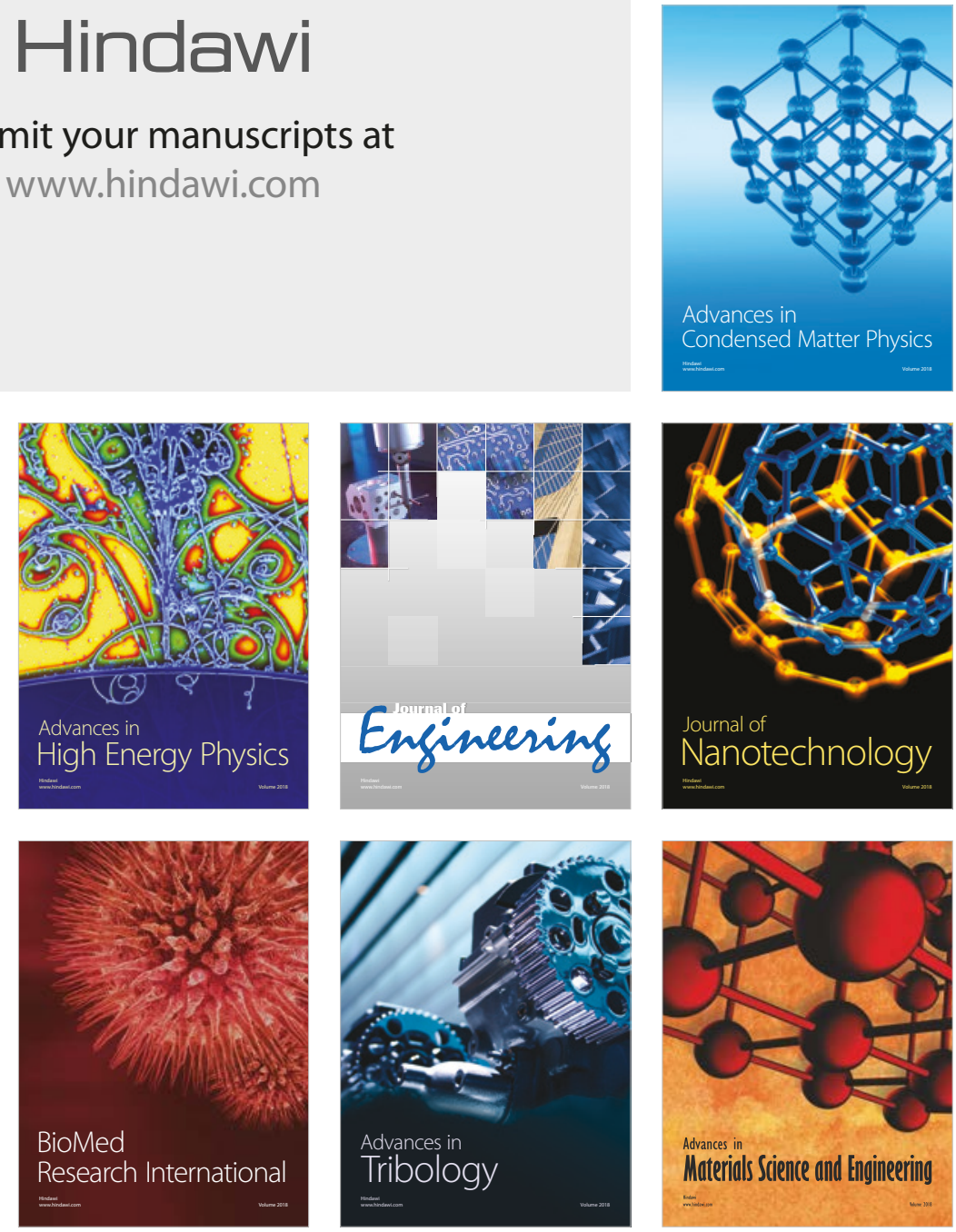